> La niche hématopoïétique médullaire est une structure pluricellulaire dont les composantes sont actuellement bien identifiées. Les cellules souches hématopoïétiques y sont notamment en contact intime avec des cellules stromales mésenchymateuses, des ostéoblastes et des cellules endothéliales sinusoïdales. Ces relations étroites sont à l'origine d'un équilibre subtil entre des fonctions cellulaires spécialisées (prolifération et quiescence, différenciation et autorenouvellement), assurant ainsi une hématopoïèse efficace. Chez les patients atteints de leucémie myéloïde chronique (LMC), affection clonale de la cellule souche hématopoïétique, des travaux récents ont montré la présence d'un dialogue entre les progéniteurs myéloïdes malins et les cellules du microenvironnement médullaire. Une niche leucémique faite de signaux altérés semble ainsi s'organiser, permettant tout à la fois une prolifération intense de cellules myéloïdes différenciées quittant la niche vers le sang périphérique et le maintien d'un pool de cellules souches leucémiques. Cette niche médullaire pourrait également protéger les cellules souches de LMC des traitements par inhibiteurs de tyrosine kinase et contribuer ainsi à leur résistance vis-àvis des thérapies ciblées. <

La leucémie myéloïde chronique (LMC) est une néoplasie myéloproliférative évoluant, en l'absence de traitement, d'une phase chronique peu symptomatique à une phase aiguë plus agressive. Malgré son incidence réduite, elle est devenue un modèle privilégié d'étude des mécanismes oncogéniques, des thérapies ciblées, de la maladie résiduelle, de l'immunité antitumorale, mais aussi, plus récemment, un modèle d'interruption thérapeutique et d'investigation de la niche hémato-

Cet article fait partie du numéro thématique publié par médecine/sciences en avril 2014 et intitulé « Microenvironnement tumoral ».

\section{Leucémie myéloïde chronique}

\section{Un modèle de dialogue entre la cellule souche leucémique et la niche hématopoïétique}

\author{
Jean-Claude Chomel ${ }^{1,2}$, Djamel Aggoune ${ }^{2}$, \\ Nathalie Sorel ${ }^{1,2}$, Ali G. Turhan ${ }^{2,3,4}$
}

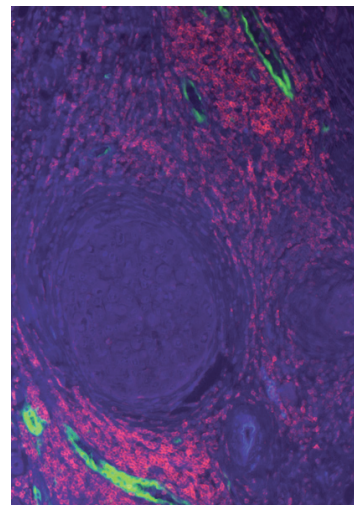

poiétique. Le chromosome Philadelphie ou Phl, correspondant à la translocation $\mathrm{t}(9 ; 22)(\mathrm{q} 34 ; \mathrm{q} 1 \mathrm{l})$, a été la première anomalie génétique décrite dans une affection maligne. Elle est à l'origine de l'oncogène chimérique BCR-ABLI (breakpoint cluster region/Abelson), qui code pour une protéine de fusion dont l'activité tyrosine kinase (produit du gène $A B L 1$ ) est dérégulée [1]. Depuis une dizaine d'années, les patients atteints de LMC bénéficient d'une thérapie ciblée particulièrement efficace basée sur l'utilisation d'inhibiteurs de tyrosine kinase (ITK). L'imatinib (Glivec $\left.{ }^{\circledR}\right)$, premier inhibiteur compétitif de l'ATP à être utilisé, cible l'oncoprotéine $B C R-A B L 1$, mais aussi les protéines kinases c-Kit et PDGF-R (platelet-derived growth factor-receptor) ${ }^{1}$. Cependant, des résistances à l'imatinib peuvent survenir, dues en partie à la présence de mutations ponctuelles au niveau du domaine kinase de BCR-ABLI. Des ITK de $2^{e}$ génération (ITK-2) comme le nilotinib (inhibiteur sélectif dérivé de l'imatinib), le dasatinib et le bosutinib (inhibiteurs SRC/ABLI) ont été développés. Ils sont plus efficaces que l'imatinib chez la plupart des patients ayant développé une résistance au Glivec ${ }^{\circledR}$, à l'exception de ceux présentant la substitution en position 315 d'une thréonine en isoleucine (T315I). Plus récemment, le ponatinib (ITK-3) s'est révélé efficace quelles que soient les mutations entraînant la résistance [2]. On dispose donc désormais d'un arsenal thérapeutique complet permettant de traiter les patients atteints de

\footnotetext{
${ }^{1}$ Cette propriété explique l'intérêt de l'imatinib dans le traitement des tumeurs stromales gastro-intestinales (GIST), caractérisées par des mutations de cKit et du PDGF-R (voir la synthèse de J.F. Emile, Med Sci 2013, vol $\left.29 n^{\circ} 6 / 7\right)$.
} 


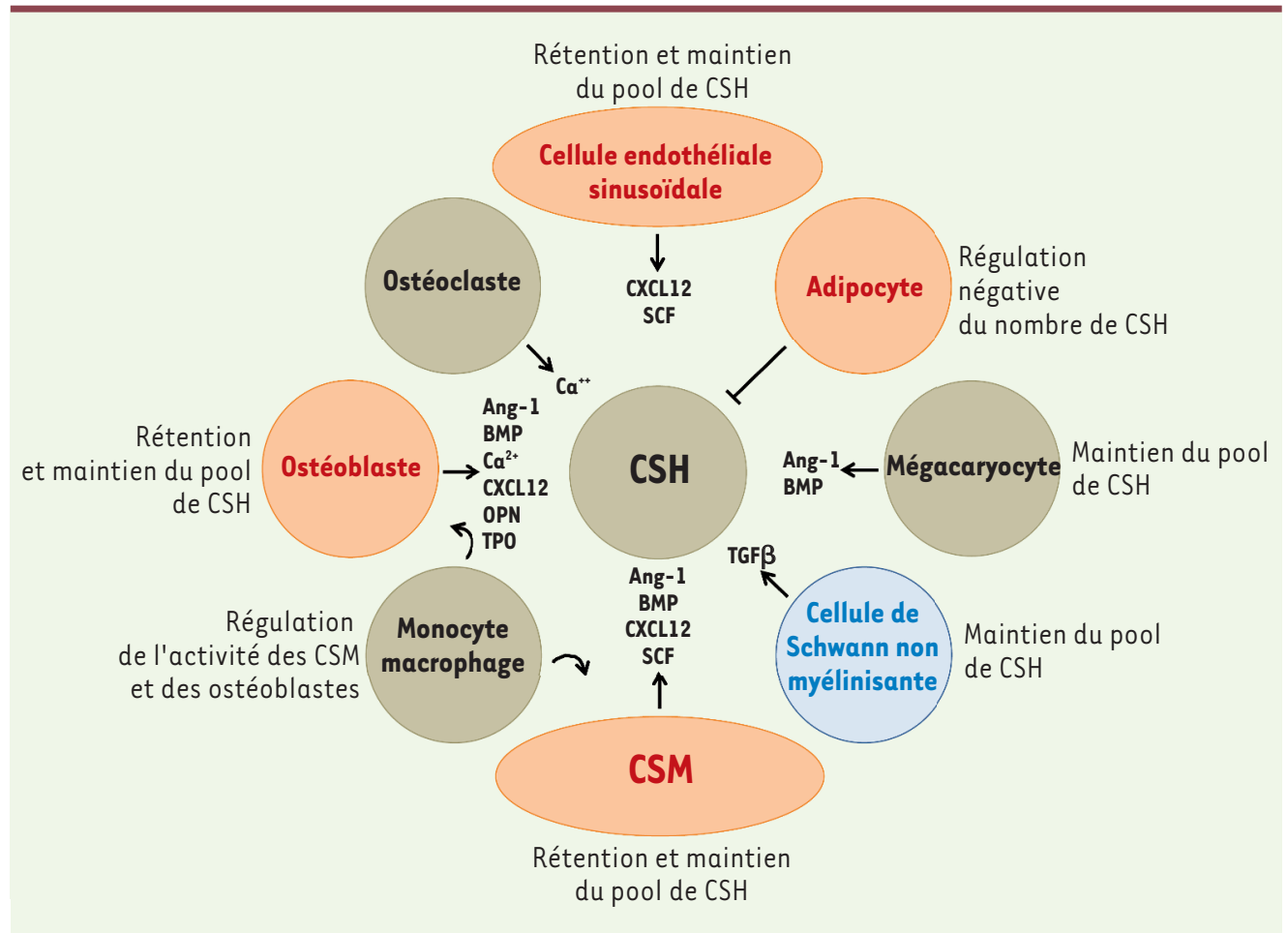

Figure 1. Représentation schématique de la niche hématopoïétique. La niche hématopoïétique de la moelle osseuse comprend des cellules d'origine mésenchymateuse (cellules stromales mésenchymateuses ou CSM, ostéoblastes, cellules endothéliales sinusoïdales, adipocytes), hématopoïétique (cellules souches hématopoïétiques ou CSH, mégacaryocytes, monocytes/macrophages, ostéoclastes) ou nerveuse (cellules de Schwann non myélinisantes). Les signaux clés de régulation des CSH par les cellules de la niche sont schématisés.
LMC avec une grande efficacité et d'adapter le traitement en fonction des intolérances ou des résistances observées. De plus, des tests moléculaires performants permettent un suivi régulier de la maladie par la quantification des $A R N m B C R-A B L 1$. Grâce aux thérapies ciblées, la plupart des patients atteints de LMC ont dorénavant une espérance de vie comparable à celle de la population générale, ce qui est exceptionnel pour une affection maligne.

Une proportion significative de patients traités par imatinib sont considérés comme étant en rémission moléculaire profonde (définie par un taux faible ou indétectable d'ARNm $B C R-A B L 1$ dans le sang périphérique) et de longue durée. La cinétique d'apparition de cette réponse, ainsi que sa profondeur, devraient être améliorées par les ITK-2. Ces résultats encourageants ont permis d'élaborer plusieurs essais d'arrêt de traitement, intéressant en premier lieu l'imatinib. Dans l'essai français STIM (stop imatinib), malgré des résultats remarquables, une rechute moléculaire (le plus souvent précoce) a été observée dans environ $60 \%$ des cas [3]. Chez de tels patients, la persistance de progéniteurs ou cellules souches leucémiques (CSL) exprimant l'ARNm $B C R-A B L 1$ a été démontrée en utilisant des tests clonogéniques et des cultures à long terme [4]. II est tentant d'expliquer la rechute moléculaire observée dans la plupart des essais d'arrêt de l'imatinib par la présence d'un réservoir de CSL quiescentes inaccessible à la thérapie ciblée. De plus, l'observation de rechutes très tardives après allogreffe de cellules souches hématopoïétiques (CSH) démontre la persistance à long terme de ces CSL. Même si toutes ces cellules souches ne sont pas forcément susceptibles d'induire une rechute leucémique, seule leur éradication devrait permettre une guérison de la LMC. Comme les CSH normales, les CSL de LMC sont présentes dans la moelle osseuse dans une structure spécialisée appelée niche hématopoïétique. Ce microenvironnement permet aux CSL non seulement d'initier une hématopoïèse leucémique (prolifération, différenciation), mais également de s'y maintenir à l'état quiescent.

\section{Microenvironnement médullaire et hématopoïèse physiologique}

L'hématopoïèse assure la production et le renouvellement continu et régulé des cellules sanguines (globules rouges, polynucléaires, monocytes, lymphocytes et plaquettes). C'est un processus fondamental capable de s'adapter en permanence à diverses conditions physiologiques, voire pathologiques. L'hématopoï̀se adulte se déroule essentiellement dans la moelle dite « rouge » (hématogène, par opposition à la moelle «jaune» graisseuse) des vertèbres, des côtes, du sternum et du bassin, ainsi que dans les épiphyses du fémur et de l'humérus. C'est un système hiérarchisé comportant à son sommet les CSH qui donnent naissance aux progéniteurs, puis aux précurseurs et enfin aux cellules différenciées. Afin d'assurer une hématopoïèse fonctionnelle tout au long de la vie, les CSH ont la capacité de s'autorenouveler ou de se différencier, d'entrer en quiescence ou de proliférer. L'équilibre entre ces différents états fonctionnels peut être contrôlé par les CSH elles-mêmes. De nombreuses voies de régulation intrinsèque sont impliquées dans l'induction de la quiescence [5]. On peut notamment citer les voies de signalisation p53, Fox0, 
Alox5a (arachidonate 5-lipoxygenase a), ATM (ataxia telangiectasia mutated, en réponse au stress oxydatif), de l'hypoxie (HIF-l $\alpha$ ), des suppresseurs de tumeurs PTEN (phosphatase and tensin homolog), PML (promyelocytic leukemia, régulateurs négatifs de mTOR [mammalian target of rapamycin]) et de STAT5 (signal transducer and activator of transcription 5). D'autres signaux intrinsèques, comme ceux impliquant les protéines BMIl et la famille des homéoprotéines Hox (HoxB4) jouent un rôle important dans le processus d'autorenouvellement [6].

\section{La niche hématopoïétique}

Les mécanismes extrinsèques impliqués dans la régulation des différents états fonctionnels des CSH découlent d'une relation étroite avec d'autres cellules ou des composants de la matrice extracellulaire au sein d'un microenvironnement particulier appelé niche hématopoïétique [7]. Le modèle de référence reste à ce jour basé sur la localisation anatomique et distingue des niches endostéales (niche hypoxique au contact de l'os) et vasculaires (ou périvasculaires) [8]. Cependant, s'agit-il de niches différentes exerçant des fonctions distinctes ou d'une niche unique associant des caractéristiques endostéales et vasculaires [9] ? Existe-t-il des niches spécialisées induisant/maintenant la quiescence ou l'autorenouvellement? Quelle que soit sa réalité, ce microenvironnement ne doit pas être considéré comme une structure figée et les différents concepts comme contradictoires. En effet, les composants cellulaires et acellulaires du microenvironnement médullaire interagissent les uns avec les autres dans le but de maintenir une hématopoïèse efficace.

\section{Les composants de la niche hématopoïétique}

La moelle osseuse est un tissu dont la vascularisation (capillaires sinusoïdes) et l'innervation (système nerveux sympathique) sont extrêmement riches. Les vaisseaux sanguins permettent l'échange de cellules et de macromolécules de la moelle osseuse vers le sang périphérique (et inversement). La niche hématopoïétique médullaire est constituée des cellules hématopoïétiques (CSH, progéniteurs, précurseurs) et de cellules stromales (cellules non hématopoïétiques). Les composants cellulaires de la niche produisent des cytokines, des chémokines ainsi qu'une matrice extracellulaire (MEC) classiquement constituée de molécules de collagène (I et IV), de laminine, de fibronectine, de protéoglycanes, de glycosaminoglycanes. Les cellules stromales du microenvironnement osseux ont une origine mésenchymateuse (cellules stromales mésenchymateuses ou CSM, cellules sinusoïdales endothéliales, ostéoblastes, adipocytes), hématopoïétique (monocytes/macrophages, ostéoclastes, mégacaryocytes), ou neurale (cellules de Schwann non myélinisantes) $[8,10]$. Ces cellules interagissent avec les CSH, par l'intermédiaire notamment de facteurs solubles (Figure 1).

Les CSM $^{2}$ constituent le composant clé de la niche hématopoïétique [10]. C'est un ensemble hétérogène de cellules dont certaines ont conservé une capacité de cellules souches (d'où leur autre

${ }^{2}$ Voir à ce propos le numéro thématique consacré par médecine/sciences aux cellules souches mésenchymateuses en mars 2011 (vol $27 \mathrm{n}^{\circ} 3$ ). dénomination de cellules souches mésenchymateuses). Elles sont à l'origine d'une différenciation adipocytaire, ostéoblastique ou endothéliale. Parmi les cellules stromales périvasculaires bordant les capillaires sinusoïdes médullaires, on trouve les cellules réticulaires (ou cellules CAR pour CXCL12 abundant reticular cells) qui sécrètent de grandes quantités de chémokine $\mathrm{CXCL12}$ ( $C-X-C$ ligand 12 ), aussi appelée SDF-l $\alpha$ (stromal cell-derived factor $l \alpha$ ), et les CSM qui sécrètent la cytokine SCF (stem cell factor ou ligand de c-Kit). Les ostéoblastes situés au niveau de l'endoste et impliqués dans le remodelage osseux (en synergie avec les ostéoclastes) représentent l'autre élément majeur de la niche hématopoiétique.

\section{Relations entre la niche hématopoïétique et les CSH}

De nombreuses molécules transmembranaires ou solubles relayent les interactions entre les cellules de la niche hématopoïétique et les CSH (Figure 2). Elles assurent la régulation des équilibres autorenouvellement/différenciation, quiescence/prolifération, survie/ apoptose et contribuent ainsi à une réponse cellulaire intégrée [11, 12]. Le Tableau / propose une représentation schématique des conséquences fonctionnelles de ces voies de signalisation extrinsèques sur l'adhérence et la migration des CSH, leur entrée en quiescence ou en autorenouvellement, ainsi que sur la régulation de la niche hématopoïétique elle-même.

Certaines voies ont été explorées en détail [10-12]. La chémokine CXCL12 est sécrétée par les cellules CAR et, dans une moindre mesure, par les ostéoblastes. II a été montré que le gradient chimiotactique ainsi créé par les cellules stromales attirait les CSH via leurs récepteurs CXCR4. L'adhérence (et donc la rétention) des CSH dans la niche hématopoïétique se fait par l'intermédiaire de sélectines, d'intégrines (LFAl [lymphocyte functionassociated antigen 1 ou $\alpha$ L $\beta 2$ ], VLA4 ou $\alpha 4 \beta 1$, VLA5 ou $\alpha 5 \beta 1$ ), de molécules d'adhérence (CD44, ICAMI [intercellular cell adhesion molecule], VCAMI [vascular cell adhesion molecule]) ou de composants de la MEC (fibronectine, acide hyaluronique). Par ailleurs, l'ostéopontine (OPN) produite par les ostéoblastes peut agir non seulement en facilitant l'adhérence des CSH au sein de la niche, mais également en régulant la quiescence. Ce processus fondamental de protection des cellules souches est favorisé par divers facteurs solubles sécrétés par le microenvironnement osseux comme la thrombopoïétine (TPO), l'angiopoïétine 1 (Ang-1) et le TGF $\beta$ (transforming growth factor $\beta$ ). L'autorenouvellement des CSH est contrôlé par plusieurs récepteurs à activité tyrosine kinase (FLT3 [Fms-like tyrosine kinase 3], FGF-R [fibroblast growth factor 


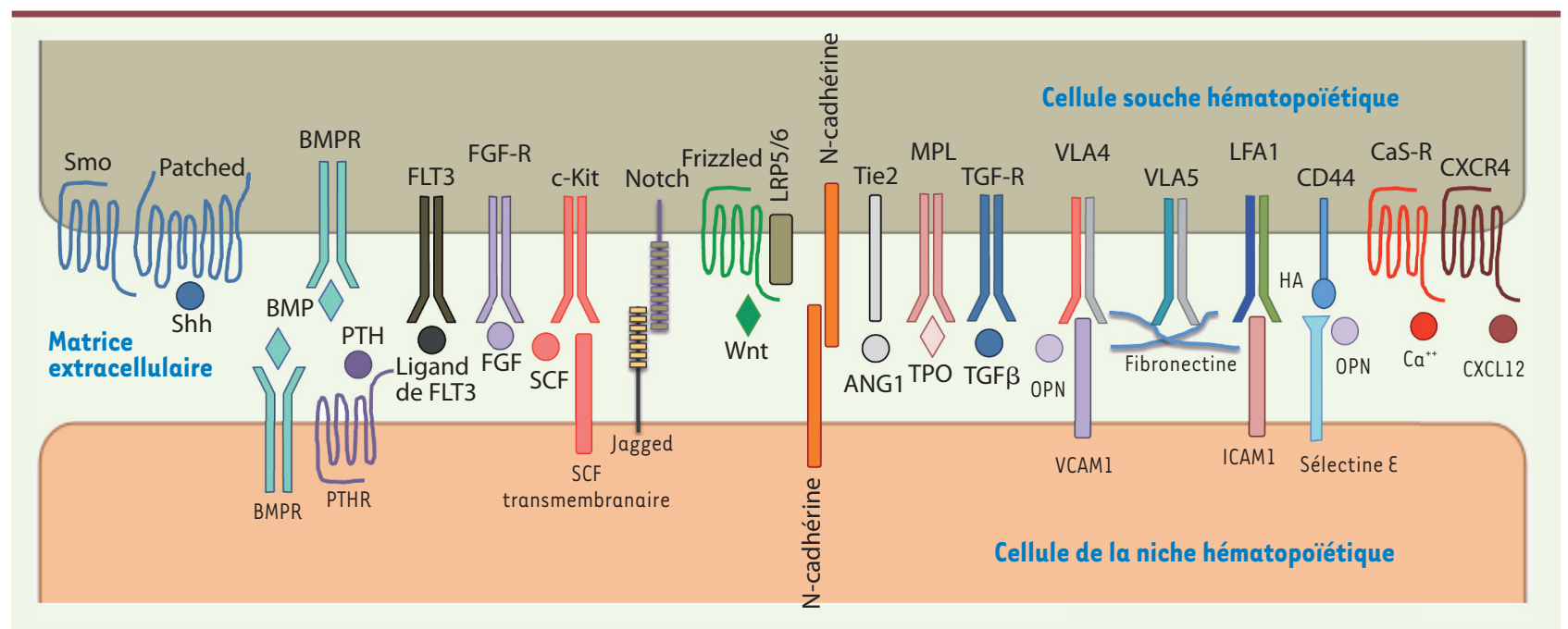

Figure 2. Les interactions CSH/niche hématopoïétique. Dans le microenvironnement osseux, les cellules souches hématopoiétiques (CSH) sont en relation étroite avec les cellules de la niche ou les composants de la matrice extracellulaire (MEC). Ces relations, ainsi que la présence de facteurs solubles sécrétés dans la MEC, jouent un rôle fondamental dans le maintien d'une hématopoïèse fonctionnelle. La figure résume les relations entre les CSH et les cellules de la niche.

receptor], c-Kit) et leurs ligands solubles ou membranaires, ainsi que par les récepteurs Notch et leur ligand Jagged. L'activation de la voie Wnt/ $\beta$-caténine influencerait l'autorenouvellement des progéniteurs immatures ou leur maintien en quiescence selon le contexte cellulaire. Enfin, la taille de la niche hématopoïétique, et, par voie de conséquence, le nombre de CSH présentes en son sein, sont notamment régulés par les protéines BMP (bone morphogenic protein) de la superfamille du TGF $\beta$, et la parathormone (PTH).

\section{Cellules souches leucémiques et niche hématopoiétique}

Les CSL de LMC sont localisées, comme les CSH normales, dans le microenvironnement osseux. Néanmoins, des résultats récents laissent penser que leurs relations avec les composants de la niche sont modifiées de manière à favoriser le maintien d'une hématopoïèse leucémique. Celle-ci contribue à la prolifération et la différenciation de cellules leucémiques exprimant le gène chimérique $B C R-A B L I$ tout en préservant le pool de CSL. De nombreuses études, basées sur des modèles cellulaires ou murins, suggèrent l'existence d'altérations dans le nichage des CSL et le dialogue que celles-ci établissent avec les cellules stromales. Nous illustrerons cette hypothèse à l'aide de quelques exemples, afin de définir les concepts de niche leucémique et de résistance thérapeutique liée à la niche, et nous examinerons les nouvelles possibilités thérapeutiques qui découlent de ces observations.

\section{Nichage, prolifération et quiescence des cellules souches de LMC}

La localisation des CSH normales au sein de la niche hématopoïétique ou nichage fait intervenir au moins trois composants: les sélectines, les intégrines et les chimioattractants. II y a plus de vingt ans, un défaut d'adhérence des progéniteurs hématopoïétiques exprimant l'oncogène $B C R-A B L I$ aux cellules stromales et à la fibronectine du microenvironnement médullaire avait été mis en évidence [13, 14]. Des anomalies fonctionnelles ou conformationnelles de la sous-unité $\beta 1$ des intégrines $\alpha \beta$ avaient alors été évoquées. Bien que toujours présentes à la surface des cellules, les intégrines VLA4 $(\alpha 4 \beta 1)$ et VLA5 $(\alpha 5 \beta 1)$ ne permettraient plus une adhérence normale des CSL au stroma médullaire. Dans la LMC, l'attraction des CSL vers le microenvironnement osseux en réponse au gradient de chémokine CXCL12 (SDF-1 $\alpha$ ) serait également réduite [15]. La voie de signalisation ou l'expression du récepteur CXCR4 seraient affectées selon le niveau d'expression de l'oncoprotéine BCR-ABL1 [16]. La perte du chimiotactisme déclenché par le gradient CXCL12 et de l'adhérence liée aux intégrines $\beta 1$ expliquerait la migration des cellules leucémiques immatures hors de la niche et donc la prolifération cellulaire exacerbée observée dans la moelle osseuse, suivie d'une mobilisation des cellules vers le sang périphérique.

Cependant, le maintien, chez les patients atteints de LMC, d'un pool de cellules souches assurant l'hématopoïèse leucémique, nécessite une voie alternative de nichage. Celle-ci ferait intervenir, spécifiquement dans les CSL exprimant $B C R-A B L 1$ et contrairement aux $C S H$ normales, l'expression de la glycoprotéine de surface CD44 [17]. L'adhérence des CSL aux cellules de la niche hématopoïétique pourrait avoir lieu via une interaction CD44/sélectines $\varepsilon$. De plus, une surexpression d'ostéopontine (pouvant se fixer sur le récepteur CD44 des CSL) a été montrée in vitro dans des cellules $B C R-A B L^{+}$et un 


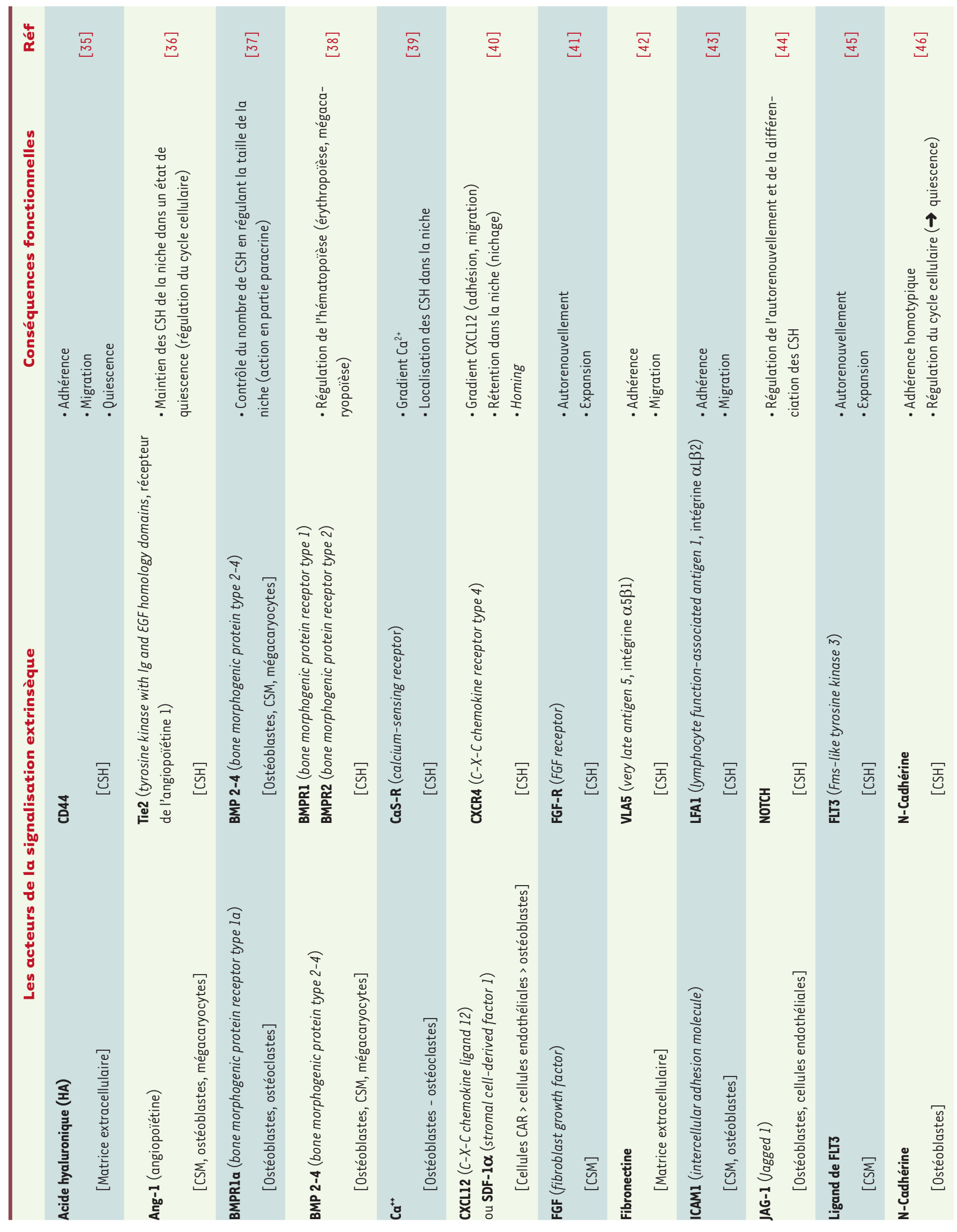




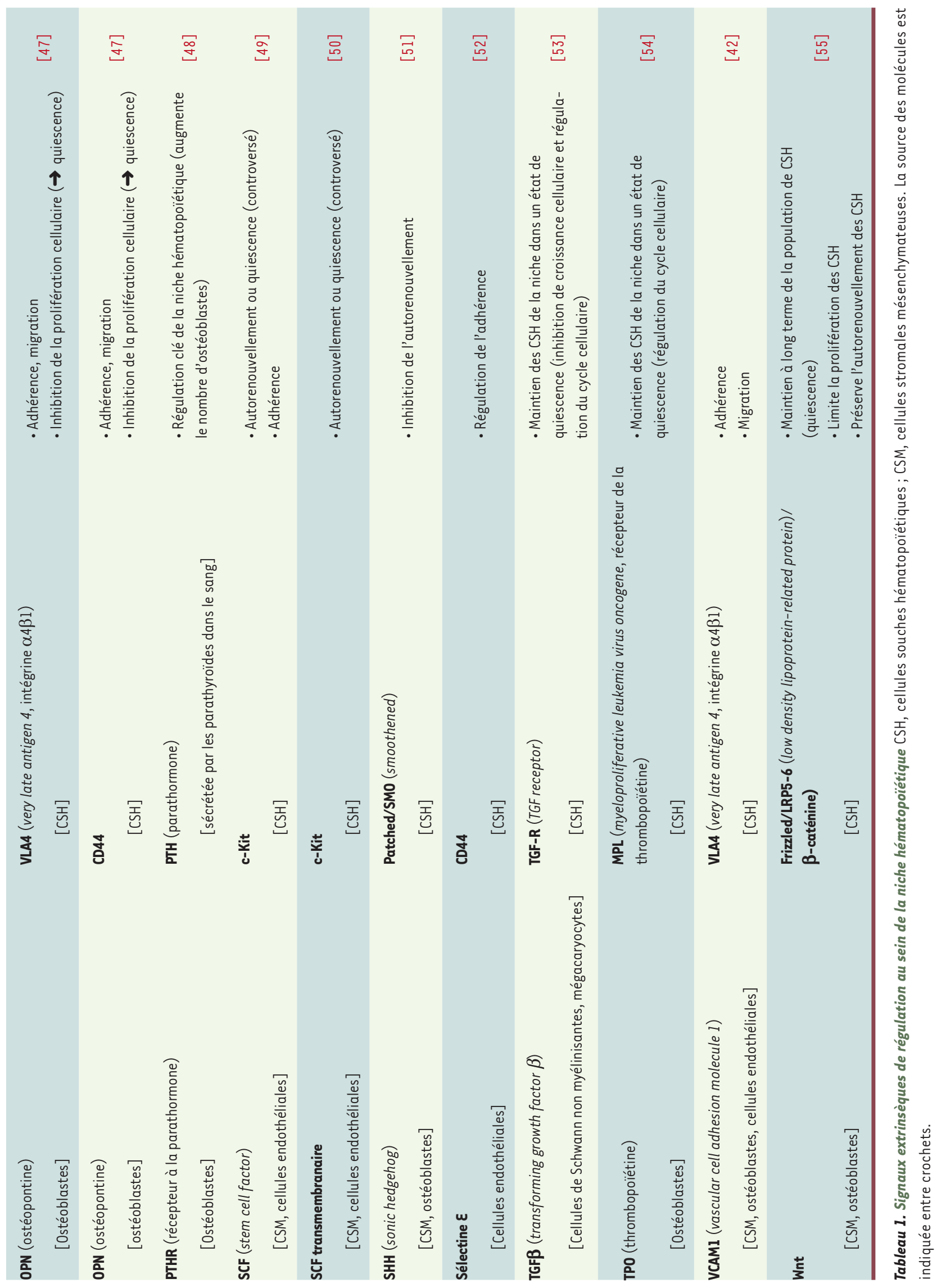


taux élevé de cette protéine a été détecté dans le sérum des patients atteints de LMC [18]. Ces données suggèrent l'existence de mécanismes de nichage propres aux CSL de LMC, leur permettant à la fois de maintenir leur quiescence au sein du microenvironnement médullaire et de proliférer de façon incontrôlée.

Il est à noter que l'interféron- $\alpha$, ainsi que la tyrphostine AG957 (inhibiteur de la kinase $B C R-A B L 1$ ), permettraient de rétablir la fonction adhésive des intégrines $\alpha n \beta l$ et donc un contrôle de la prolifération hématopoïétique $[19,20]$. Lors du traitement par imatinib, la réponse au gradient $\mathrm{CXCL12}$ serait partiellement restaurée; les CSL résiduelles pourraient alors être attirées (comme les CSH normales) dans le microenvironnement osseux qui les protégerait en induisant des processus de survie et de quiescence [21].

\section{CSH de LMC et niche hématopoïétique : un dialogue modifié}

Le dialogue (cross-talk) entre CSH et cellules du microenvironnement médullaire est indispensable au maintien d'une hématopoïèse physiologique. Chez les patients atteints de LMC, plusieurs travaux ont suggéré que ce dialogue pouvait être altéré au profit des cellules leucémiques.

Ainsi, en étudiant un modèle de souris transgénique exprimant de façon inductible l'oncogène $B C R-A B L 1$, Schepers et al. ont montré qu'une interaction directe entre les cellules leucémiques myéloïdes et les CSM était à l'origine d'un remodelage de la niche ostéoblastique [22]. Sous l'influence des cellules leucémiques et de facteurs solubles comme la TPO, les CSM seraient alors conduites à donner naissance à un nombre élevé de cellules ostéoblastiques anormales. Ce phénomène permettrait la prolifération et la différenciation des cellules leucémiques, mais défavoriserait le maintien du pool de CSH normales via une diminution d'expression de facteurs impliqués dans la rétention ou la quiescence (CXCL12, SCF, Ang-1). Le fait que le pool de CSL soit épargné pourrait s'expliquer par la mise en jeu par ces dernières d'autres systèmes de rétention et d'adhérence au microenvironnement médullaire [17]. Ainsi, l'hématopoïèse leucémique serait favorisée au détriment de l'hématopoïèse normale. D'autres exemples du dialogue CSL-niche hématopoïétique ont récemment été rapportés, démontrant l'importance de ce phénomène dans les mécanismes de leucémogenèse.

Le PIGF (placental growth factor) est un facteur de croissance de la famille du VEGF (vascular endothelial growth factor). Schmidt et al. ont montré que les cellules de la niche pouvaient produire du PIGF sous l'influence de lignées cellulaires exprimant $B C R-A B L 1$ [23]. Ce mécanisme serait dépendant de la voie NF- $\kappa B$ et nécessiterait une liaison VLA4-VCAMI entre les cellules leucémiques et les CSM. Ces travaux ont par ailleurs montré des concentrations élevées de PIGF dans le sang et la moelle osseuse de patients atteints de LMC. Le PIGF pourrait agir, d'une part sur le microenvironnement osseux par une activation des mécanismes d'angiogenèse et par une accumulation de CSM, et, d'autre part, sur les cellules leucémiques en facilitant leur prolifération [23]. Il créerait ainsi, au sein du microenvironnement osseux, un terrain proleucémique fertile caractérisé par une vascularisation importante.
La galectine-3 ( $\mathrm{Gal}-3)$ est une lectine qui est le plus souvent sécrétée par les macrophages; elle joue un rôle dans l'adhérence cellulaire, la migration, la prolifération et l'apoptose. Des études en cocultures ont montré la production de Gal-3 par plusieurs lignées humaines $B C R-A B L^{+}$sous l'influence de cellules stromales HS- 5 [24]. De plus, des taux élevés de Gal-3 ont été détectés dans les cellules leucémiques isolées de la moelle osseuse de patients atteints de LMC. Dans ces conditions, la production de Gal-3 serait à l'origine d'une prolifération in vitro des cellules leucémiques et de leur résistance vis-à-vis d'agents thérapeutiques (activation des voies de signalisation AKT et $\varepsilon R K$, accumulation du facteur antiapoptotique Mcll). Enfin, en utilisant un modèle de xénogreffes chez des souris immunodéficientes, les auteurs ont montré que la galectine-3 faciliterait le nichage des CSL.

Les protéines BMP ont un rôle majeur dans les différents mécanismes régulant les $\mathrm{CSH}$, notamment dans I'autorenouvellement et la différenciation. Au cours de la LMC, il a été récemment observé une augmentation des taux de protéines BMP2 et BMP4 solubles dans la niche hématopoïétique, ainsi qu'une hyperexpression du récepteur BMPRIb par les CSL [25]. Ces modifications, identifiées aussi bien dans des lignées cellulaires que dans des cellules primaires isolées de patients au moment du diagnostic, seraient présentes dès la phase chronique de la maladie et auraient pour conséquence une expansion de la lignée myéloïde (BMP 2-4), ainsi que le maintien d'un pool de CSL (BMP4).

\section{Concept de niche leucémique}

Les données expérimentales décrites ci-dessus suggèrent donc fortement l'existence d'échanges réciproques entre les progéniteurs de LMC et leur microenvironnement médullaire. Ce dialogue modifierait les fonctions principales de la niche hématopoïétique afin non seulement de promouvoir l'expansion de cellules malignes, mais aussi de préserver un pool de CSL (Figure 3). Existe-t-il alors une nouvelle niche spécialisée, la niche leucémique, permettant aux cellules souches $B C R-A B L^{+}$de persister et de proliférer? Les résultats des travaux exposés ci-dessus semblent montrer que le concept de niche leucémique est une réalité dont il faut désormais tenir compte dans les stratégies d'éradication de la LMC $[22,25]$. Le traitement par ITK entraîne chez une majorité de patients une rémission moléculaire. Dans ces conditions, nous avons vu que les interactions entre les cellules leucémiques et les composants de la niche pouvaient encore être modifiées en favorisant la persistance d'un pool résiduel de cellules 


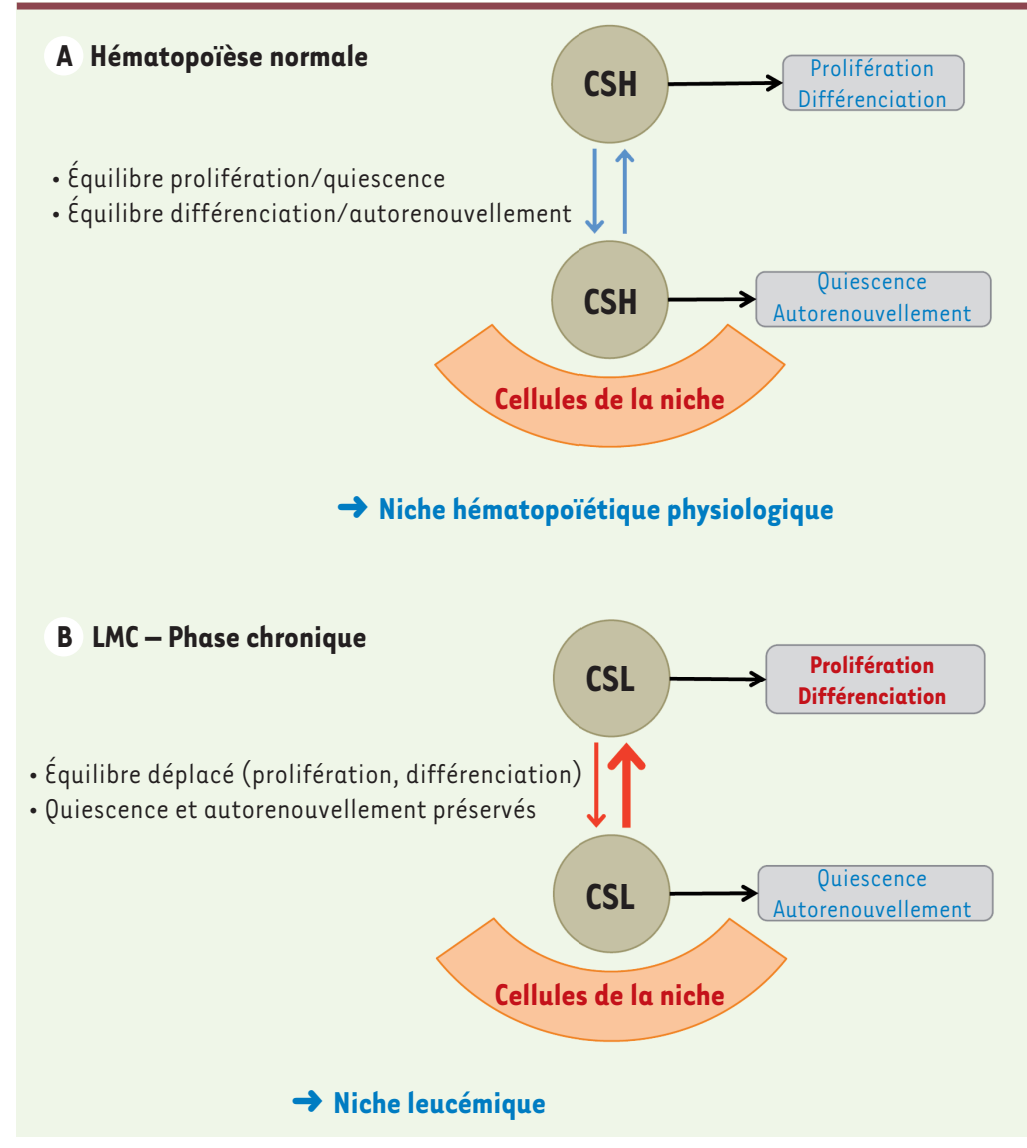

Figure 3. Modèle de niche leucémique. Les cellules souches leucémiques (CSL) de LMC entament un dialogue avec les cellules de la niche hématopoïétique. Cet échange serait à l'origine d'une véritable niche leucémique permettant la prolifération et la différenciation des cellules myéloïdes malignes tout en maintenant un pool de CSL et défavorisant l'hématopoïèse normale.

CSL résiduelles de la niche afin de les rendre à nouveau sensibles aux ITK (Figure 4B). Pour être réellement intéressants, les éventuels traitements ciblant la niche devraient être les plus spécifiques possibles afin de ne pas toucher l'hématopoïèse normale.

La solution de Fowler (à base d'arsénite de potassium) a été utilisée dans le traitement des leucémies dès le $x x^{\mathrm{e}}$ siècle et fut la première drogue à montrer une certaine efficacité chez des patients atteints de LMC [29]. Des expériences de coculture de cellules K562 (une lignée érythroleucémique dérivée d'un patient atteint de LMC) avec des cellules stromales (issues de la moelle osseuse de patients atteints de LMC) ont renouvelé l'intérêt thérapeutique $d u$ trioxyde d'arsenic [30]. Celui-ci induirait une diminution de l'expression des intégrines $\beta 1$ à souches de LMC. Ainsi, au début de la maladie (phase chronique), la niche hématopoïétique serait altérée par les cellules leucémiques et acquérerait les caractéristiques d'une niche anormale promouvant la prolifération et la différenciation de cellules myéloïdes malignes tout en préservant les CSL. Après traitement par ITK et obtention d'une rémission moléculaire, la niche serait à nouveau remodelée pour devenir une niche de quiescence des CSL (Figure 4A). La résistance aux ITK pourrait donc être paradoxalement induite par l'inhibiteur luimême (notion de résistance liée à la niche). Le microenvironnement médullaire modifié par les cellules leucémiques constituerait ainsi un sanctuaire pour des CSL résiduelles [26-28]. La persistance d'un pool de CSL quiescentes au sein d'une niche hématopoïétique devenue complice représente une épée de Damoclès pour le patient, puisque ces cellules peuvent à tout moment entrer en cycle et réinitialiser le mécanisme de leucémogenèse.

\section{Une thérapie spécifique de la niche leucémique}

Les traitements actuels de la LMC ciblent les cellules leucémiques et plus particulièrement l'activité tyrosine kinase dérégulée de BCR$A B L 1$. L'éradication complète de la maladie passe en théorie par l'élimination des CSL présentes au sein de la niche leucémique. On peut donc tenter de cibler les mécanismes de régulation de l'hématopoïèse, qu'ils soient intrinsèques (Fox $0, \mathrm{PML}, \mathrm{Alox} 5 \mathrm{a}$ ) ou extrinsèques (liés à la niche leucémique). L'une des options serait de déloger les la surface des cellules K562, ce qui réduirait leur adhérence au support stromal et les rendrait sensibles au traitement cytotoxique.

Le plérixafor (ou AMD 3100) est un antagoniste réversible du récepteur CXCR4, empêchant la fixation de la chémokine CXCL12. Dans les protocoles d'autogreffes, ce produit peut être utilisé, en association avec du G-CSF (granulocyte-colony stimulating factor), afin de mobiliser les CSH de la moelle osseuse vers le sang périphérique où elles pourront être prélevées. Dans le cas de la LMC, I'utilisation combinée de plérixafor et d'un ITK pourrait déloger les CSL du microenvironnement médullaire et les rendre sensibles à la thérapie ciblée. Les résultats de cette association thérapeutique, testée dans des modèles murins, sont cependant controversés $[31,32]$.

Les anticorps anti-PIGF peuvent prévenir ou traiter une angiogenèse pathologique, notamment dans le cadre des affections cancéreuses [33]. Dans le cas de la LMC, I'inhibition du PIGF pourrait avoir un effet additif à celui de l'imatinib [23]. Enfin, plusieurs autres cibles peuvent être envisagées comme la galectine-3 (utilisation du complexe polysaccharidique GCS-100 bloquant les effets de $\mathrm{Gal}-3$ ) et l'ostéopontine (utilisation d'anticorps anti-OPN) [24, 34]. 


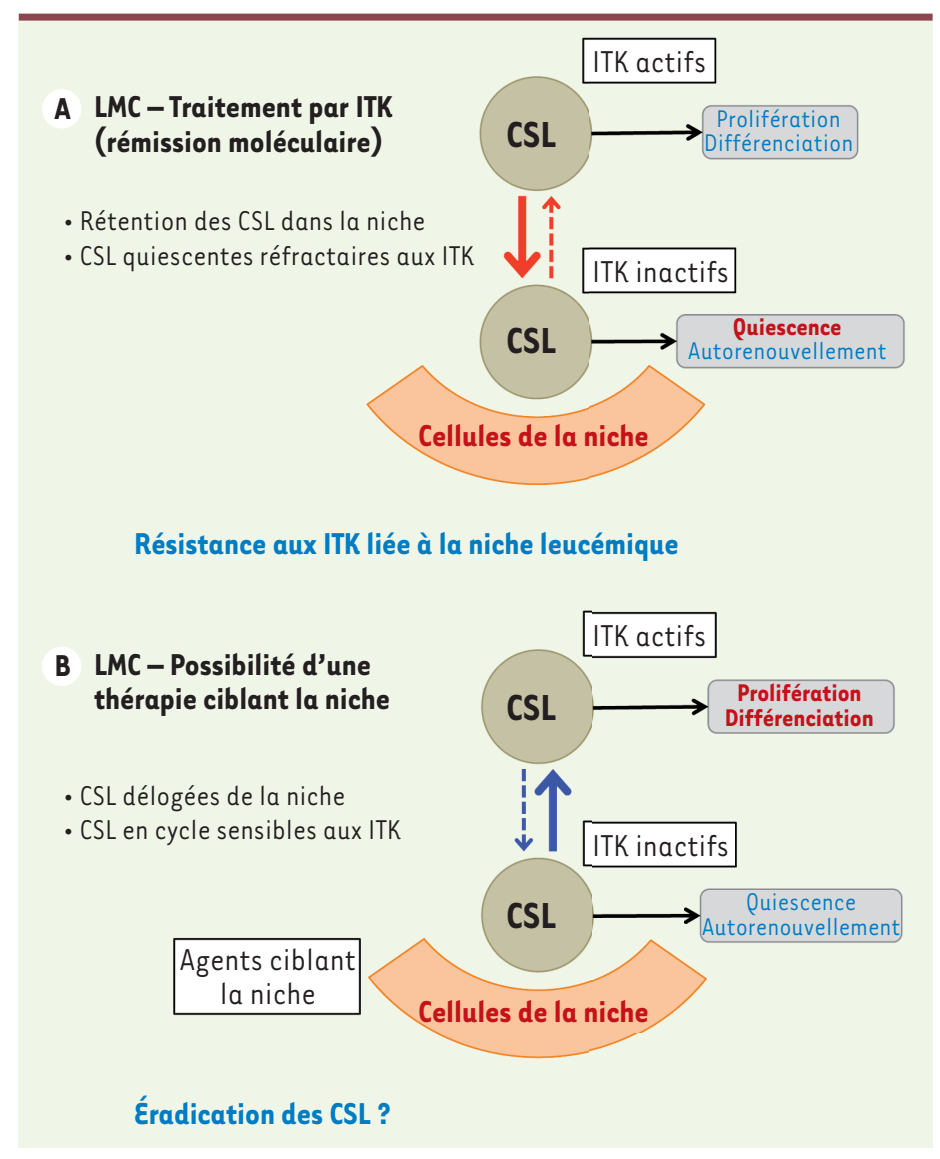

\section{Conclusion}

Les patients atteints de LMC traités en première ligne par les ITK et présentant une réponse favorable ont désormais une espérance de vie similaire à celle d'une population indemne de la maladie. Les thérapies ciblées permettent d'obtenir des rémissions moléculaires profondes et de longue durée, autorisant des essais d'interruption de traitement. Malgré des résultats remarquables, la guérison de la maladie est pour l'instant difficile à affirmer parce qu'il est nécessaire de poursuivre le traitement à long terme chez la très grande majorité des patients et que persiste un contingent de CSL quiescentes et résistantes aux ITK, protégées dans une niche devenue leur sanctuaire. Une meilleure compréhension des relations intercellulaires au sein du microenvironnement médullaire devrait faciliter le développement de drogues ciblant les cellules souches de LMC ou des éléments de la niche leucémique. Un traitement combinant un ITK et une thérapie ciblant la niche pourrait permettre de faire un pas décisif vers la guérison de la LMC. $\diamond$

\section{SUMMARY}

Chronic myeloid leukemia stem cells: cross-talk with the niche

The physiological hematopoietic niche located in bone marrow is a pluricellular structure whose components are now well identified. Within this microenvironment, hematopoietic stem cells are in direct contact with mesenchymal stromal cells, osteoblasts and sinusoidal endothelial cells. These close relationships drive specialized cellular
Figure 4. Inhibiteurs de tyrosine kinase et niche leucémique. Ce modèle présente les relations potentielles entre les cellules souches leucémiques (CSL) et la niche dans un contexte de traitement par inhibiteurs de tyrosine kinase (ITK). Les cellules souches leucémiques résiduelles migrent vers la niche. Des mécanismes intrinsèques et extrinsèques (émanant de la niche) favorisent le maintien des progéniteurs leucémiques en phase de quiescence. Ces cellules deviennent alors résistantes aux ITK. Des traitements ayant pour objectif de déloger les CSL de la niche leucémique permettraient alors leur éradication.

functions (proliferation/quiescence, differentiation/ self-renewal) ensuring an efficient hematopoiesis. Chronic myeloid leukemia (CML) is a major model of leukemic hematopoiesis. The BCR-ABLI tyrosine kinase, constitutively activated in CML, plays a critical role in the pathogenesis of the disease. An intensive cross-talk between CML progenitors and the components of the hematopoietic niche has recently been demonstrated. Consequently, the occurrence of the so-called leukemic niche promotes both the proliferation of myeloid cells and the maintenance of quiescent leukemic stem cells. This bone marrow niche could also protect CML stem cells from tyrosine kinase inhibitors and probably contribute to their resistance towards targeted therapies. $\diamond$

\section{LIENS D'INTÉRÊT}

Les auteurs déclarent n'avoir aucun lien d'intérêt concernant les données publiées dans cet article.

\section{RÉFÉRENCES}

1. Wong S, Witte $O N$. The BCR-ABL story: bench to bedside and back. Annu Rev Immunol $2004 ; 22: 247-306$.

2. Baccarani M, Deininger MW, Rosti G, et al. European LeukemiaNet recommendations for the management of chronic myeloid leukemia: 2013. Blood 2013; $122: 872-84$.

3. Mahon FX, Rea D, Guilhot J, et al. Discontinuation of imatinib in patients with chronic myeloid leukaemia who have maintained complete molecular remission for at least 2 years: the prospective, multicentre Stop Imatinib (STIM) trial. Lancet Oncol $2010 ; 11: 1029-35$.

4. Chomel JC, Bonnet ML, Sorel N, et al. Leukemic stem cell persistence in chronic myeloid leukemia patients with sustained undetectable molecular residual disease. Blood $2011 ; 118: 3657-60$.

5. Li L, Bhatia R. Stem cell quiescence. Clin Cancer Res 2011 ; 17 : 4936-41.

6. Faubert A, Chagraoui J, Mayotte N, et al. Complementary and independent function for Hoxb4 and Bmil in HSC activity. Cold Spring Harb Symp Quant Biol $2008 ; 73: 555-64$.

7. Schofield R. The relationship between the spleen colony-forming cell and the haemopoietic stem cell. Blood Cells $1978 ; 4: 7-25$.

8. Lataillade JJ, Brunet de La Grange P, Uzan G, Le Bousse-Kerdiles MC. Les cellules souches ont-elles l'âge de leur niche ? À la recherche d'un sérum de jouvence. Med Sci (Paris) $2010 ; 26: 582-5$.

9. Kiel MJ, Morrison SJ. Uncertainty in the niches that maintain haematopoietic stem cells. Nat Rev Immunol $2008 ; 8: 290-301$.

10. Frenette PS, Pinho S, Lucas D, Scheiermann C. Mesenchymal stem cell: keystone of the hematopoietic stem cell niche and a stepping-stone for regenerative medicine. Annu Rev Immunol 2013 ; 31 : 285-316. 


\section{RÉFÉRENCES}

11. Rizo A, Vellenga $\varepsilon$, de Haan $G$, Schuringa JJ. Signaling pathways in self-renewing hematopoietic and leukemic stem cells: do all stem cells need a niche? Hum Mol Genet 2006; 15 (suppl 2) R210-9.

12. Wilson A, Trumpp A. Bone-marrow haematopoietic-stem-cell niches. Nat Rev Immunol 2006 ; 6 : 93-106.

13. Gordon MY, Dowding CR, Riley GP, et al. Altered adhesive interactions with marrow stroma of haematopoietic progenitor cells in chronic myeloid leukaemia. Nature $1987 ; 328$ : 342-4.

14. Verfaillie CM, McCarthy JB, McGlave PB. Mechanisms underlying abnormal trafficking of malignant progenitors in chronic myelogenous leukemia. Decreased adhesion to stroma and fibronectin but increased adhesion to the basement membrane components laminin and collagen type IV. J Clin Invest $1992 ; 90: 1232-41$.

15. Salgia R, Quackenbush $\varepsilon$, Lin J, et al. The BCR/ABL oncogene alters the chemotactic response to stromal-derived factor-lalpha. Blood $1999 ; 94: 4233-46$.

16. Geay JF, Buet D, Zhang $Y$, et al. p210BCR-ABL inhibits SDF- 1 chemotactic response via alteration of CXCR4 signaling and down-regulation of CXCR4 expression. Cancer Res $2005 ; 65$ : 2676-83.

17. Krause DS, Lazarides K, von Andrian UH, Van Etten RA. Requirement for CD44 in homing and engraftment of BCR-ABL-expressing leukemic stem cells. Nat Med $2006 ; 12$ : 1175-80.

18. Flamant $S$, Kortulewski T, Dugray $A$, et al. Osteopontin is upregulated by BCR-ABL. Biochem Biophys Res Commun 2005 ; 333 : 1378-84.

19. Bhatia R, McCarthy JB, Verfaillie CM. Interferon-alpha restores normal beta 1 integrin-mediated inhibition of hematopoietic progenitor proliferation by the marrow microenvironment in chronic myelogenous leukemia. Blood 1996 ; 87 : 3883-91.

20. Bhatia R, Munthe HA, Verfaillie CM. Tyrphostin AG957, a tyrosine kinase inhibitor with anti-BCR/ $A B L$ tyrosine kinase activity restores betal integrin-mediated adhesion and inhibitory signaling in chronic myelogenous leukemia hematopoietic progenitors. Leukemia 1998 ; 12 : 1708-17.

21. Jin L, Tabe Y, Konoplev S, et al. CXCR4 up-regulation by imatinib induces chronic myelogenous leukemia $(\mathrm{CML})$ cell migration to bone marrow stroma and promotes survival of quiescent $\mathrm{CML}$ cells. Mol Cancer Ther $2008 ; 7: 48-58$.

22. Schepers K, Pietras EM, Reynaud D, et al. Myeloproliferative neoplasia remodels the endostea bone marrow niche into a self-reinforcing leukemic niche. Cell Stem Cell $2013 ; 13: 285-99$.

23. Schmidt T, Kharabi Masouleh B, Loges $\mathrm{S}$, et al. Loss or inhibition of stromal-derived PIGF prolongs survival of mice with imatinib-resistant Bcr-Abll(+) leukemia. Cancer Cell 2011; 19:740-53.

24. Yamamoto-Sugitani M, Kuroda J, Ashihara $\varepsilon$, et al. Galectin-3 (Gal-3) induced by leukemia microenvironment promotes drug resistance and bone marrow lodgment in chronic myelogenous leukemia. Proc Natl Acad Sci USA 2011 ; 108 : 17468-73.

25. Laperrousaz B, Jeanpierre S, Sagorny K, et al. Primitive CML cell expansion relies on abnorma levels of BMPs provided by the niche and on BMPRIb overexpression. Blood $2013 ; 122$ : 3767-77.

26. Nair RR, Tolentino J, Hazlehurst LA. The bone marrow microenvironment as a sanctuary for minimal residual disease in CML. Biochem Pharmacol $2010 ; 80: 602-12$.

27. Nwajei F, Konopleva M. The bone marrow microenvironment as niche retreats for hematopoietic and leukemic stem cells. Adv Hematol 2013 ; 2013 : 953982.

28. Krause DS, Scadden DT, Preffer FI. The hematopoietic stem cell niche: home for friend and foe? Cytometry B Clin Cytom $2013 ; 84$ : 7-20.

29. Forkner C $\varepsilon$, Scott TF. Arsenic as a therapeutic agent in chronic myelogenous leukemia. JAMA 1931 ; $97: 3-5$.

30. Guo-Bao W, Xiao-Pin C, Qi-Rong G, et al. Arsenic Trioxide overcomes cell adhesion-mediated drug resistance through down-regulating the expression of betal-integrin in K562 chronic myelogenous leukemia cell line. Leuk Lymphoma 2010 ; 51 : 1090-7.

31. Agarwal A, Fleischman AG, Petersen CL, et al. Effects of plerixafor in combination with BCR-ABL kinase inhibition in a murine model of CML. Blood 2012 ; $120: 2658-68$

32. Weisberg $\varepsilon$, Azab AK, Manley PW, et al. Inhibition of CXCR4 in CML cells disrupts their interaction with the bone marrow microenvironment and sensitizes them to nilotinib. Leukemia $2012 ; 26$ : 985-90.

33. Galaup A, Germain S. Les anticorps anti-PIGF. Un nouvel outil thérapeutique anti-angiogénique? Med Sci (Paris) $2008 ; 24: 459-62$.

34. Boyerinas B, Zafrir M, Yesilkanal AE, et al. Adhesion to osteopontin in the bone marrow niche regulates lymphoblastic leukemia cell dormancy. Blood 2013; 121:4821-31.

35. Nilsson SK, Haylock DN, Johnston HM, et al. Hyaluronan is synthesized by primitive hemopoietic cells, participates in their lodgment at the endosteum following transplantation, and is involved in the regulation of their proliferation and differentiation in vitro. Blood $2003 ; 101: 856-62$.
36. Arai F, Hirao A, Ohmura M, et al. Tie2/angiopoietin-1 signaling regulates hematopoietic stem cell quiescence in the bone marrow niche. Cell 2004 118 : 149-61.

37. Zhang J, Niu C, Ye L, et al. Identification of the haematopoietic stem cell niche and control of the niche size. Nature $2003 ; 425: 836-41$.

38. Bhatia M, Bonnet D, Wu D, et al. Bone morphogenetic proteins regulate the developmental program of human hematopoietic stem cells. J Exp Med $1999 ; 189: 1139-48$

39. Adams GB, Chabner KT, Alley IR, et al. Stem cell engraftment at the endosteal niche is specified by the calcium-sensing receptor. Nature 2006 439 : 599-603.

40. Sugiyama T, Kohara H, Noda M, Nagasawa T. Maintenance of the hematopoietic stem cell pool by CXCL12-CXCR4 chemokine signaling in bone marrow stromal cell niches. Immunity $2006 ; 25: 977-88$.

41. Itkin T, Kaufmann KB, Gur-Cohen S, et al. Fibroblast growth factor signaling promotes physiological bone remodeling and stem cell self-renewal. Curr Opin Hematol $2013 ; 20: 237-44$

42. Teixido J, Hemler ME, Greenberger JS, Anklesaria P. Role of beta 1 and beta 2 integrins in the adhesion of human CD34hi stem cells to bone marrow stroma. J Clin Invest $1992 ; 90: 358-67$.

43. Pruijt JF, van Kooyk Y, Figdor CG, et al. Anti-LFA-1 blocking antibodies prevent mobilization of hematopoietic progenitor cells induced by interleukin-8. Blood 1998; $91:$ 4099-105.

44. Mancini SJ, Mantei N, Dumortier A, et al. Jaggedl-dependent Notch signaling is dispensable for hematopoietic stem cell self-renewal and differentiation. Blood $2005 ; 105: 2340-2$.

45. Kikushige $\mathrm{Y}$, Yoshimoto G, Miyamoto T, et al. Human Flt3 is expressed at the hematopoietic stem cell and the granulocyte/macrophage progenitor stages to maintain cell survival. J Immunol 2008 ; 180 : 7358-67.

46. Arai F, Hosokawa K, Toyama H, et al. Role of $\mathrm{N}$-cadherin in the regulation of hematopoietic stem cells in the bone marrow niche. Ann NY Acad Sci 2012 ; $1266: 72-7$.

47. Nilsson SK, Johnston HM, Whitty GA, et al. Osteopontin, a key component of the hematopoietic stem cell niche and regulator of primitive hematopoietic progenitor cells. Blood $2005 ; 106: 1232-9$.

48. Calvi LM, Adams GB, Weibrecht KW, et al. Osteoblastic cells regulate the haematopoietic stem cell niche. Nature 2003 ; 425 : 841-6.

49. Kovach NL, Lin N, Yednock T, et al. Stem cell factor modulates avidity of alpha 4 beta $l$ and alpha 5 beta $l$ integrins expressed on hematopoietic cell lines. Blood $1995 ; 85$ : 159-67.

50. Driessen RL, Johnston HM, Nilsson SK. Membrane-bound stem cell factor is a key regulator in the initial lodgment of stem cells within the endosteal marrow region. Exp Hematol 2003 ; 31 : 1284-91.

51. Bhardwaj G, Murdoch B, Wu D, et al. Sonic hedgehog induces the proliferation of primitive human hematopoietic cells via BMP regulation. Nat Immunol $2001 ; 2: 172-80$.

52. Dimitroff CJ, Lee Jy, Rafii S, et al. CD44 is a major $\varepsilon$-selectin ligand on human hematopoietic progenitor cells. J Cell Biol $2001 ; 153$ : 1277-86.

53. Yamazaki S, Ema H, Karlsson G, et al. Nonmyelinating Schwann cells maintain hematopoietic stem cell hibernation in the bone marrow niche. Cell $2011 ; 147: 1146-58$

54. Yoshihara H, Arai F, Hosokawa K, et al. Thrombopoietin/MPL signaling regulates hematopoietic stem cell quiescence and interaction with the osteoblastic niche. Cell Stem Cell $2007 ; 1:$ 685-97.

55. Reya T, Duncan AW, Ailles $L$, et al. A role for Wnt signalling in self-renewal of haematopoietic stem cells. Nature 2003 ; 423 : 409-14.

\section{TIRÉS À PART}

J.-C. Chomel 